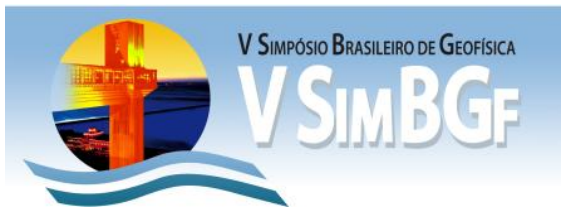

\title{
Otimização empírica de coeficientes na parametrização por série ondaleta Haar de campos de velocidades sísmicas
}

Alexsandro G. Cerqueira*, UFBA, Salvador BA, Brasil

Wilson M. Figueiró, CPGG-IGEO-UFBA, Salvador, BA, Brasil

Copyright 2012, SBGf - Sociedade Brasileira de Geofísica

Este texto foi preparado para a apresentação no V Simpósio Brasileiro de Geofísica, Salvador, 27 a 29 de novembro de 2012. Seu conteúdo foi revisado pelo Comitê Técnico do V SimBGf, mas não necessariamente representa a opinião da SBGf ou de seus associados. É proibida a reprodução total ou parcial deste material para propósitos comerciais sem prévia autorização da SBGf.

\section{Abstract}

General and theoretical aspects of wavelet series are presented, particularly those on the Haar wavelet. This is a special type of such series. A two-dimensional (2D) function is represented by an one-dimensional (1D) Haar wavelet series. Two 2D compressional seismic velocity fields derived from known geological models (the Angular Discordance with a porous layer satured with hydrocarbon and the Horst of Recôncavo) are parameterized using a strategy that allow to represent a 2D function by means of a 1D Haar wavelet series. The objective of this work is to optimize empirically the number of coefficients of the Haar wavelet series, used in the representation of the mentioned models, by means of high-pass filters in order to suppress coefficients of smallamplitude (zero or near zero).

Keywords: Haar Wavelet Series, Parameterization, Seismic Velocity Field, and High-Pass Filter.

\section{Resumo}

Aspectos gerais e teóricos das séries ondaleta são apresentados, em particular aqueles sobre a ondaleta Haar. Esta é um tipo especial de tais séries. Uma função bidimensional (2D) é representada pela série ondaleta Haar. Dois campos 2D de velocidades sísmicas compressionais derivados de modelos geológicos conhecidos (Discordância Angular com camada porosa saturada com hidrocarboneto e 0 do Horst do Recôncavo) são parametrizados usando uma estratégia que permite representar uma função $2 \mathrm{D}$ através de uma serie ondaleta Haar 1D. O objetivo do trabalho é otimizar de forma empírica a quantidade de coeficientes da série ondaleta Haar, usada na representação dos modelos citados, através de filtros passa-alta para suprimir coeficientes de pequena amplitude (nulos ou quasenulos).

Palavras chaves: Série Ondaleta Haar, Parametrização, Campo de Velocidades Sísmicas e Filtro Passa-Alta.

\section{Introdução}

Um problema matemático de grande interesse, não apenas em termos teóricos, mas também com respeito a aplicações, é aquele relativo à representação (ou decomposição) de funções complicadas como uma combinação linear (série) de funções simples ou de fácil manipulação. Como alguns exemplos mais conhecidos de tais séries, temos: Taylor, Fourier, Splines, e etc. Todas possuem qualidade e defeitos. Uma nova alternativa surgiu e se desenvolveu no último século e vem encontrando grandes aplicações na representação e na compressão de dados. Trata-se da série ondaleta (wavelet series), que se mostra apropriada na representação de funções causais (que possuem integral finita de seu módulo). Um caso particular, simples e mais antigo de tais séries é aquela conhecida por ondaleta Haar, cuja base de funções consiste em dilatações (ou contrações), translações, e superposições das funções caixa.

Utilizando a ferramenta matemática que é a ondaleta, é possível representar funções contínuas e não contínuas por uma combinação linear de funções pertencentes a uma base, cuja resolução pode ser alterada a partir de parâmetros (coeficientes) que participam na forma matemática (série) na qual participam os elementos da base ondaleta utilizada.

Esses parâmetros são o foco do estudo desse trabalho, tendo em vista que o objetivo é diminuir o número de coeficientes utilizados para representar tais modelos tentando manter a qualidade dos mesmos.

Para que fosse feita a representação de tais campos de velocidades sísmicas por série ondaleta, assim como a filtragem de seus coeficientes: foram necessárias diferentes estratégias algébricas e numéricas, assim como, a criação de diferentes algoritmos, os quais foram testados computacionalmente para comprovar a validade das representações dos campos de velocidade.

\section{Conceitos Teóricos Básicos}

A base ondaleta é definida como aquela constituída por funções do tipo: $\left(\psi_{j, k}\right)$, chamadas de ondaletas filhas, geradas a partir de uma dilatação binária $2^{\mathrm{j}}$ e uma translação diádica $k \cdot 2^{-j}$ de uma função $\psi$, chamada de função ondaleta (ou ondaleta-mãe), onde j e $k \in Z$. A idéia é considerar dilatações, compressões e translações 
dessa única função $\psi$ de modo a obter uma aproximação a mais exata possível da função que se deseja representar. Essa base de funções obedece à seguinte forma (Morettin, 1999):

$\psi_{j, k}(t)=\psi\left(2^{j} t-k\right)$, onde $j$ e $k \in Z$.

Essas funções formam uma base que não precisa ser necessariamente ortogonal, sendo que, é mais vantajoso trabalhar com bases ortogonais, para que seja possível uma reconstrução perfeita do sinal original, pois, cada coeficiente é calculado como o produto interno do sinal com uma função da base.

Tendo-se por interesse bases de ondaletas que sejam ortogonais, a Eq. (1) pode ser reescrita como:

$\psi_{j, k}(t)=2^{j / 2} \cdot \psi\left(2^{j} t-k\right)$, onde $j$ e $k \in Z$.

O sistema ortonormal $\left(\psi_{j, k}\right)$ é uma base de $L^{2}(R)$, espaço das funções reais de variável real quadrado integráveis. Existem coeficientes $\mathrm{c}_{\mathrm{j}, \mathrm{k}}$ que tornam possível representar uma função $f(t)$ da seguinte forma:

$f(t)=\sum_{j=-\infty}^{\infty} \sum_{k=-\infty}^{\infty} c_{j, k} \cdot \psi_{j, k}(t)$.

A equação (3) é denominada série ondaletas e seus coeficientes são dados por:

$c_{j, k}=\left\langle f \mid \psi_{j, k}\right\rangle=\int_{-\infty}^{\infty} f(t) \cdot \psi_{j, k}(t) d t$.

Vale ressaltar que a relação de Parseval,

$\int_{-\infty}^{\infty} f^{2}(t) d t=\sum_{j} \sum_{k} c_{j, k}^{2}$

também é valida na análise ondaletas.

A função $\psi$ pode apresentar algumas propriedades, tais como:

P1) $\int_{-\infty}^{\infty} \psi(t) d t=0$ (admissibilidade),

P2) $\int_{-\infty}^{\infty}|\psi(t)| d t<\infty$,

P3) $\int_{-\infty}^{\infty} \frac{|\widehat{\psi}(\omega)|^{2}}{|\omega|} d \omega<\infty, \hat{\psi}(\omega)$ é a transformada de Fourier de $\psi(t)$ e

P4) Os primeiros $\mathrm{r}-1$ momentos de $\psi$ se anulam, isto é, $\int_{-\infty}^{\infty} t^{j} \cdot \psi(t) d t=0, j=0,1 \ldots r-1$.

Como nem todas as ondaletas geram sistemas ortogonais, faz-se necessário gerar ondaletas através de uma função escala (ou ondaleta pai), $\phi(t)$, que soluciona a equação:

$\phi(\mathrm{t})=\sqrt{2} \sum_{k} l_{k} \phi(2 \mathrm{t}-\mathrm{k})$,

onde

$l_{k}=\sqrt{2} \int_{-\infty}^{\infty} \phi(\mathrm{t}) \phi(2 \mathrm{t}-\mathrm{k}) \mathrm{dt}$.
Logo, pode-se gerar a seguinte família ortogonal em $L^{2}(R)$ :

$\phi(\mathrm{t})=2^{\frac{j}{2}} \phi\left(2^{j}-k\right)$, onde $j$ e $k \in Z$.

O sistema ortonormal:

$\left\{\phi_{j, k}(t), \psi_{j, k}(t)\right.$, onde $j$ e $\left.k \in Z\right\}$

permite reescrever $f(t)$ da seguinte forma:

$f(t)=\sum_{k} d_{j_{0}, k} \cdot \phi_{j_{0}, k}(t)+\sum_{j \geq j_{0}} \sum_{k} c_{j, k} \cdot \psi_{j, k}(t)$,

cujos coeficientes são dados por:

$c_{j, k}=\int_{-\infty}^{\infty} f(t) \cdot \psi_{j, k}(t) d t$

e

$d_{j_{0}, k}=\int_{-\infty}^{\infty} f(t) \cdot \phi_{j_{0}, k}(t) d t$.

O caso mais simples de ondaleta é a denominada de Haar, que possui expressão numérica explícita de caráter simétrico e é escrita da seguinte forma:

$\psi^{H}(t)= \begin{cases}+1, & 0 \leq t<1 / 2 \\ -1, & 1 / 2 \leq t<1 \\ 0, & \text { outros casos. }\end{cases}$

Pode-se, então, obter a base ondaleta partindo-se da Eq. (13), que fica:

$\psi_{j, k}^{H}(t)=\left\{\begin{array}{c}2^{j / 2}, \text { se } 2^{-j} k \leq t<2^{-j}(k+1 / 2) \\ -2^{j / 2}, \text { se } 2^{-j}(k+1 / 2) \leq t<2^{-j}(k+1) \\ 0, \text { outros casos. }\end{array}\right.$

O gráfico da Eq. (13) é mostrado na Figura 1.

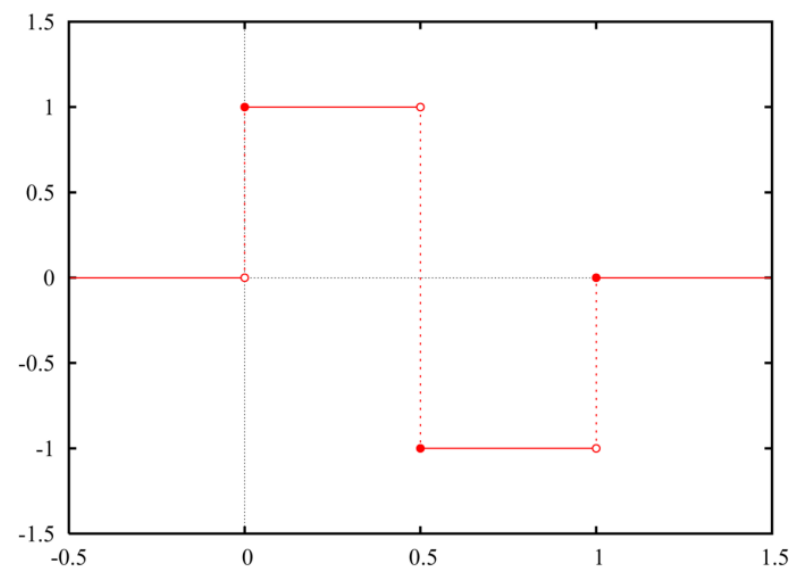

Figura 1 - Representação gráfica da função ondaleta Haar indicada na Eq. (13).

Neste trabalho, foi utilizada a Eq. (10) para realizar as representações dos modelos de campos de velocidades sísmicas, que é uma função utilizada para representar funções 1D. Entretanto, os modelos de campos de velocidades são 2D. Visando solucionar tal problema, foi 
criada uma malha senoidal para tornar possível a representação de tais campos $2 \mathrm{D}$ por funções $1 \mathrm{D}$ e assim poder utilizar a Eq. (10). Logo, os pontos desta malha relacionam-se da seguinte forma:

$z=\cos (n x)$.

Assim, pode-se representar as velocidades sísmicas, $v(x, z)$, apenas em função de $\mathrm{x}$.

O filtro utilizado é o do tipo passa-alta, cuja função é anular todos os coeficientes que tiverem valores menores que $10^{p}$, onde a escolha de $p$ tem por finalidade controlar o ponto de corte dos coeficientes.

\section{Resultados}

\section{Modelo da Sela}

Testes iniciais foram realizados com o algoritimo, onde, ao invés de usar campos de velocidades, foram utilizadas funções 2D quaisquer, como, por exemplo, a sela representada na Figura 2.

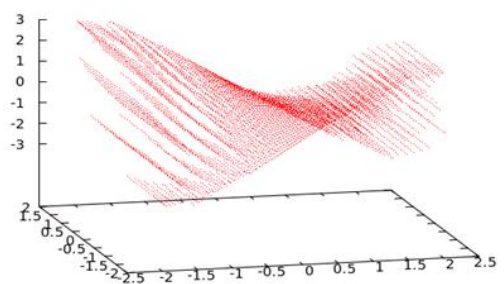

Figura 2 - Representação por parametrização utilizando a série ondaleta Haar da função sela: $f(x, y)=x \cdot y$.

\section{Modelo de Discordância Angular}

A Figura 3 mostra o modelo geológico de uma discordância angular. Extraiu-se de tal modelo a propriedade da velocidade de ondas compressionais (Figura 4) e buscou-se representá-la pela série ondaleta Haar (Figuras: 5, 6, 7, 8, 9, 10 e 11) visto que, o algoritmo de representação por série dada pela Eq. 10 funciona como o esperado. Tais modelos parametrizados foram obtidos tomando-se como referência o modelo mostrado na Figura 4.

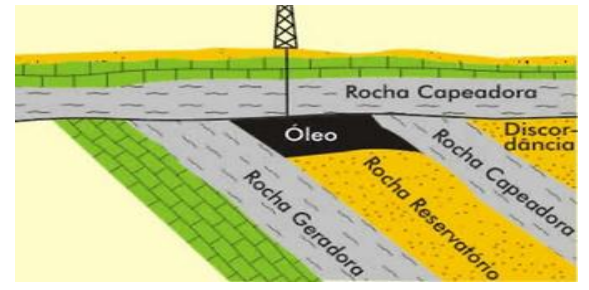

Figura 3 - Modelo geológico da Discordância Angular com acumulação de hidrocarbonetos em camada porosa (Teixeira et al., 2000).

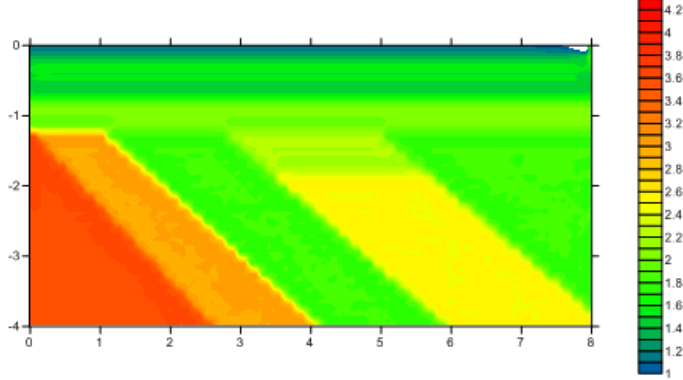

Figura 4 - Modelo numérico $\left(M_{I}\right)$ da Discordância Angular com acumulação de hidrocarboneto em camada porosa utilizado na geração dos modelos parametrizados.

A Figura 5 mostra o modelo parametrizado por série ondaleta Haar que representa o modelo numérico do campo de velocidade advindo do modelo geológico. Para tanto os índices $j$ e $k$ assumiram os seguintes valores: $j_{0}=0, j_{\text {max }}=15, k_{\text {min }}=0$ e $k_{\text {max }}=1500$. Pode-se observar na Figura 5 que as principais feições estruturais do campo podem ser, em geral, identificadas.

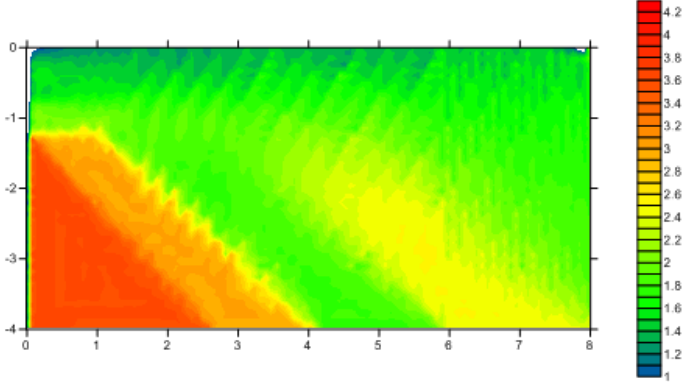

Figura 5 - Modelo $M_{I}$ parametrizado por ondaleta Haar, sem aplicação de filtros, utilizando os seguintes índices: $j_{0}=0, j_{\max }=15, k_{\min }=0$ e $k_{\max }=1500$.

Após verificar que os valores escolhidos de $j$ e $k$ representavam bem, através do modelo parametrizado, o modelo numérico, foi implementado um filtro passa-alta, do tipo $10^{p}$, para anular coeficientes menores que esse valor. $\mathrm{O}$ valor de $p$ foi variado para que fosse possível criar uma relação empírica entre a acurácia da representação e o número de coeficientes e assim escolher um modelo parametrizado que melhor representasse 0 original, mantendo o máximo de qualidade e utilizando o menor número possível de coeficientes. As Figuras de 6 à 11 mostram os modelos parametrizados obtidos através de filtragens de coeficientes. 


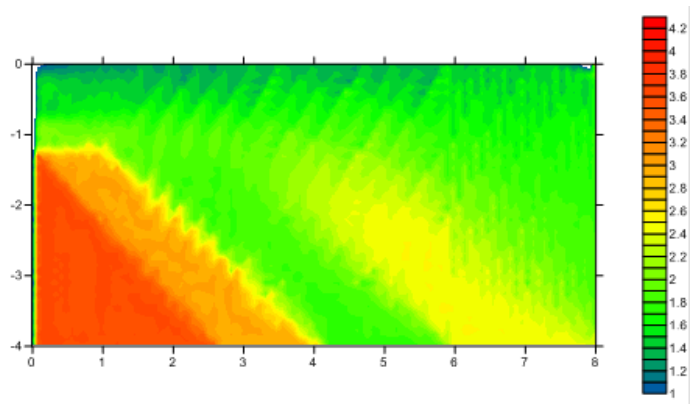

Figura 6 - Modelo $M_{I}$ parametrizado por ondaleta Haar utilizando-se o filtro passa-alta com ponto de corte igual a $10^{-3}$.
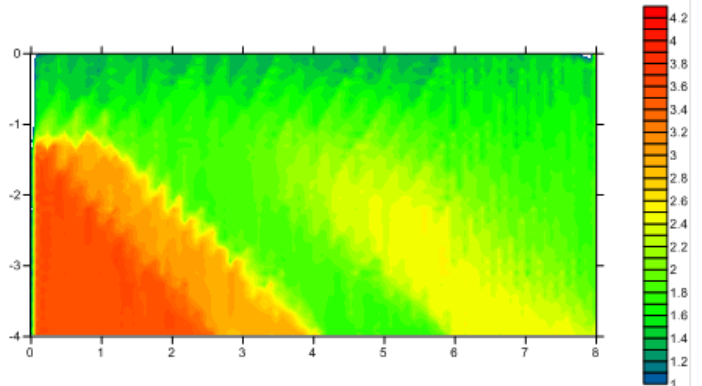

Figura 7 - Modelo $M_{I}$ parametrizado por ondaleta Haar utilizando-se o filtro passa-alta com ponto de corte igual a $10^{-2}$.

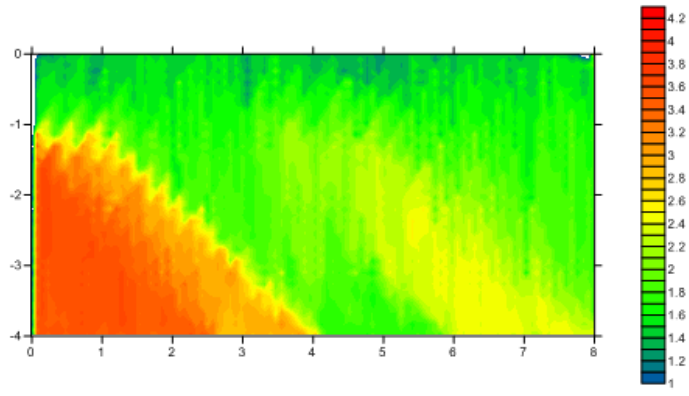

Figura 8 - Modelo $M_{I}$ parametrizado por ondaleta Haar utilizando-se o filtro passa-alta com ponto de corte igual a $10^{-1,75}$

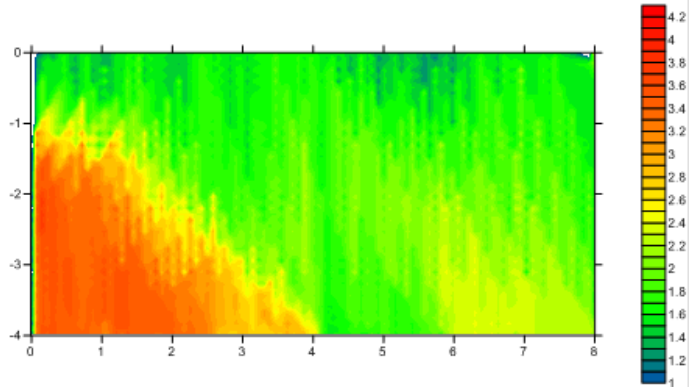

Figura 9 - Modelo $M_{I}$ parametrizado por ondaleta Haar utilizando-se o filtro passa-alta com ponto de corte igual a $10^{-1,5}$.

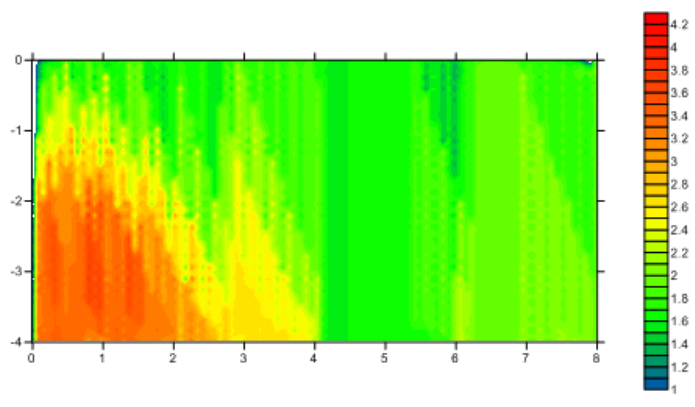

Figura 10 - Modelo $M_{I}$ parametrizado por ondaleta Haar utilizando-se o filtro passa-alta com ponto de corte igual a $10^{-1,25}$.
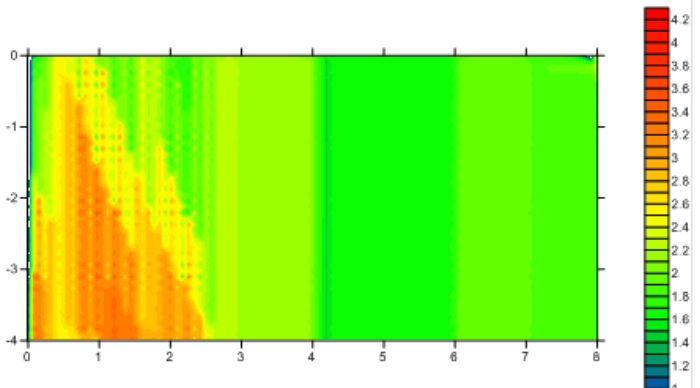

Figura 11 - Modelo $M_{I}$ parametrizado por ondaleta Haar utilizando-se o filtro passa-alta com ponto de corte igual a $10^{-1}$.

Após geradas as imagens, foi criado um gráfico que mostra, empiricamente, o grau de influência do ponto de corte do filtro na acurácia e no número de coeficientes usados na parametrização. Tal gráfico é mostrado na Figura 12.

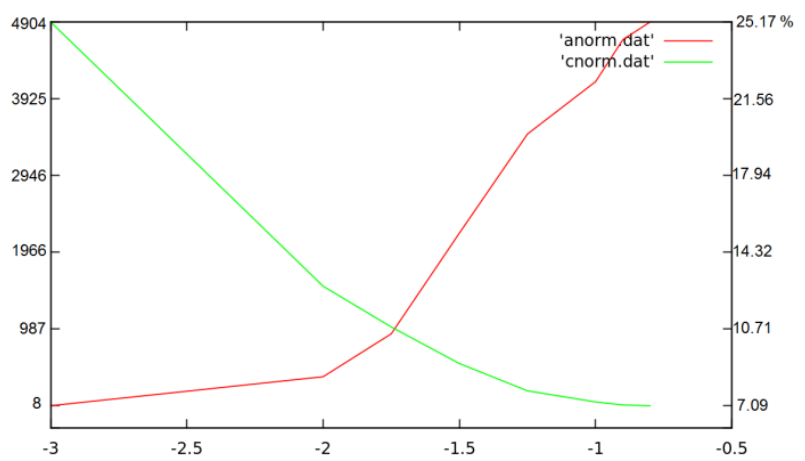

Figura 12 - Grau de influência do ponto de corte do filtro na acurácia (vermelho) e no número de coeficientes (verde) usados na parametrização de $M_{I}$. O eixo horizontal representa o expoente $p$ do ponto de corte $10^{p}$.

A técnica utilizada para determinar o ponto de corte ótimo foi aquela permitida pela interseção das duas curvas, que neste caso ocorreu no ponto $p=-1,75$, ou seja, o resultado ótimo foi aquele mostrado na Figura 8 , para a qual o ponto de corte do filtro utilizado foi $10^{-1,75}$. 
Portanto, este foi o resultado que melhor representa o campo utilizando-se a menor quantidade de coeficientes (980, aproximadamente).

\section{Modelo do Horst}

Repetindo-se o procedimento, para o modelo geológico do Horst do Recôncavo (Figura 13), foi construído o campo numérico de velocidades sísmicas compressionais (Figura 14) utilizado na geração dos modelos parametrizados por ondaleta Haar.

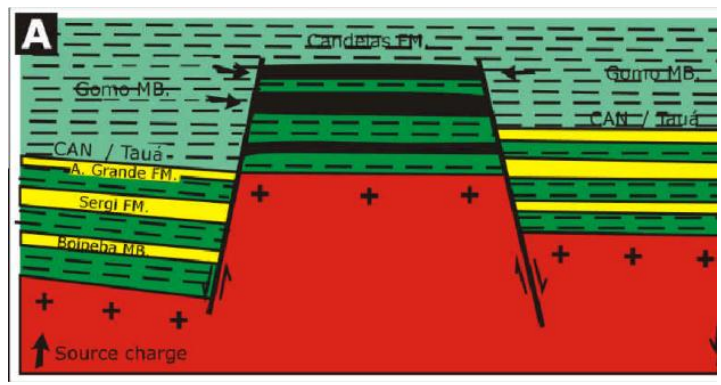

Figura 13 - Modelo geológico do Horst relativo à Trapa do Recôncavo (Santos \& Braga, 1989. In Araripe, 2002).
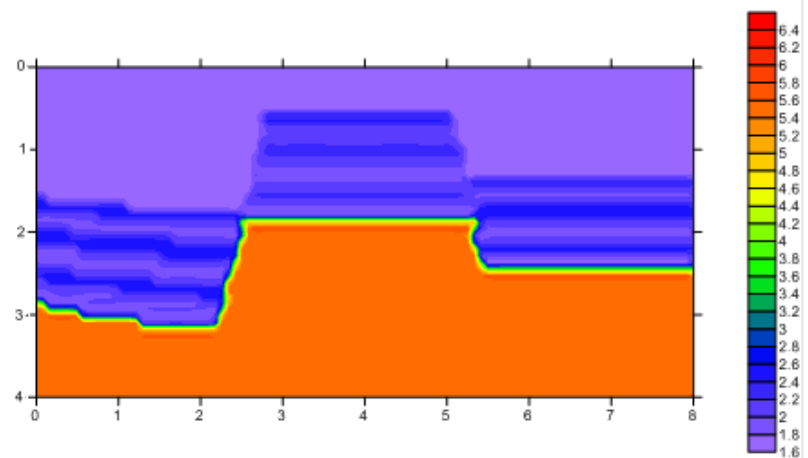

Figura 14 - Campo de velocidades numérico $\left(M_{I I}\right)$ advindo do modelo geológico do Horst, usado na geração dos modelos parametrizados.

Após gerado o modelo numérico que é representado na Figura 14, foi possível aplicar o filtro passa-alta, gerando assim as Figuras de 15 à 20.

Analogamente ao caso apresentado no modelo anterior, foi construído, de modo empírico e numérico, um gráfico (Figura 21) no qual é possível observar os comportamentos da acurácia na representação do campo de velocidades e do número de coeficientes usados, em função do ponto de corte do filtro.

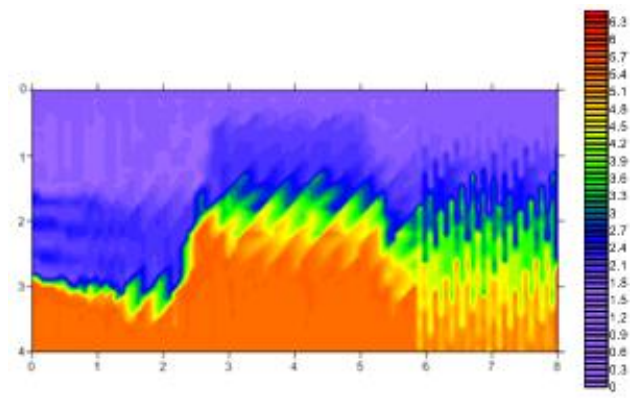

Figura 15 - Modelo $M_{I I}$ parametrizado por ondaleta Haar utilizando-se o filtro passa-alta com ponto de corte igual a $10^{-3}$.

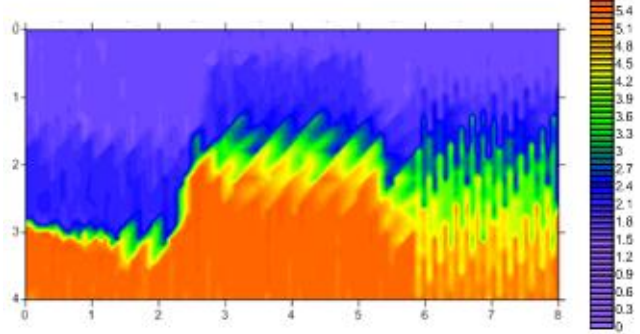

Figura 16 - Modelo $M_{I I}$ parametrizado por ondaleta Haar utilizando-se o filtro passa-alta com ponto de corte igual a $10^{-2}$.

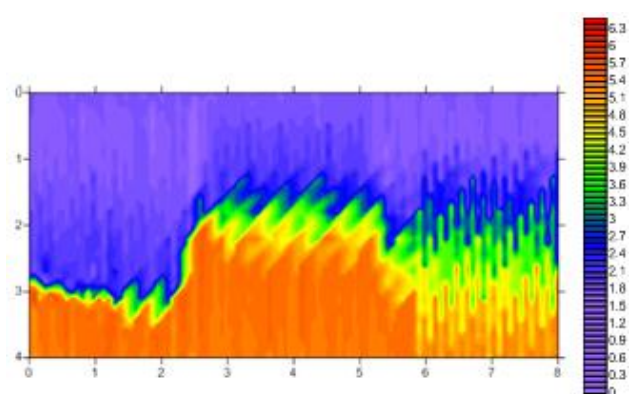

Figura 17 - Modelo $M_{I I}$ parametrizado por ondaleta Haar utilizando-se o filtro passa-alta com ponto de corte igual a $10^{-1,75}$.

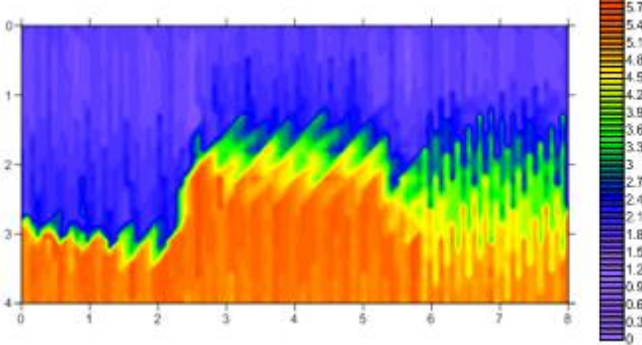

Figura 18 - Modelo $M_{I I}$ parametrizado por ondaleta Haar utilizando-se o filtro passa-alta com ponto de corte igual a $10^{-1,5}$. 


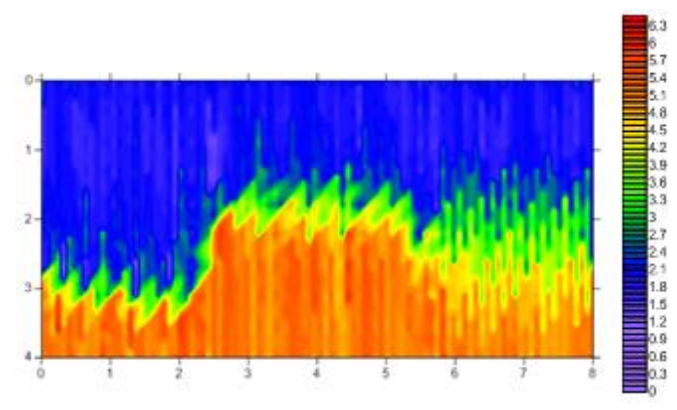

Figura 19 - Modelo $M_{I I}$ parametrizado por ondaleta Haar utilizando-se o filtro passa-alta com ponto de corte igual a $10^{-1,25}$

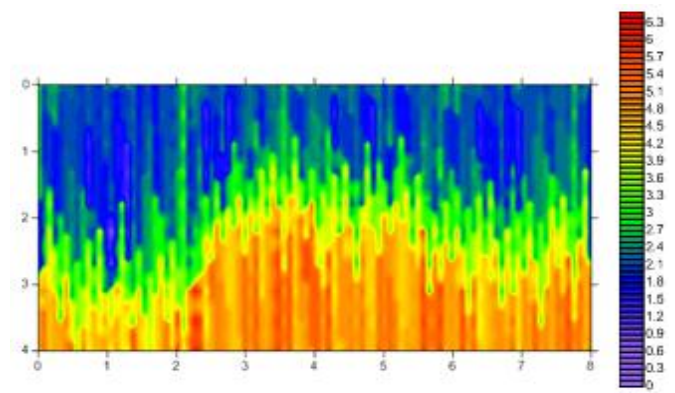

Figura 20 - Modelo $M_{I I}$ parametrizado por ondaleta Haar utilizando-se o filtro passa-alta com ponto de corte igual a $10^{-1}$.

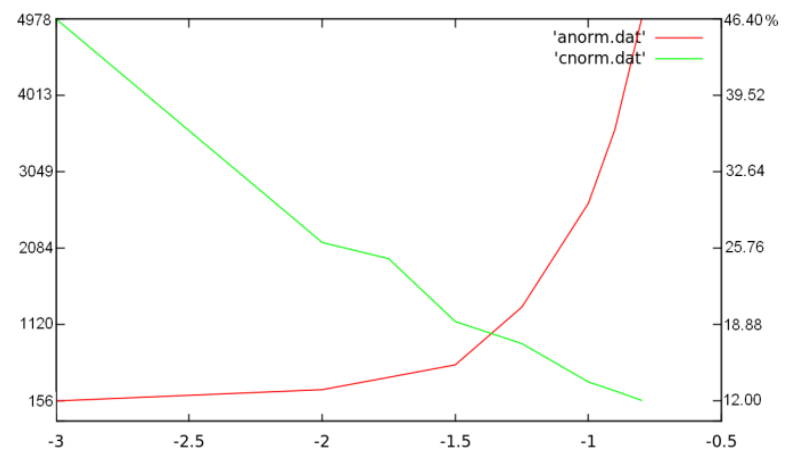

Figura 21 - Grau de influência do ponto de corte do filtro na acurácia (vermelho) e no número de coeficientes (verde) usados na parametrização de $M_{I I}$. $O$ eixo horizontal representa o expoente $p$ do ponto de corte $10^{p}$. $O$ ponto relativo à interseção das curvas refere-se a $p=-1,35$.

Aplicando-se novamente a técnica empírica que foi utilizada no modelo anterior, agora, no do Horst da Trapa do Recôncavo, observou-se que, neste caso, a interseção caiu no ponto $p=-1,35$. Ou seja, o resultado ótimo foi aquele para o qual tal valor de $p$ foi aplicado, que pode ser visto na Figura 22.

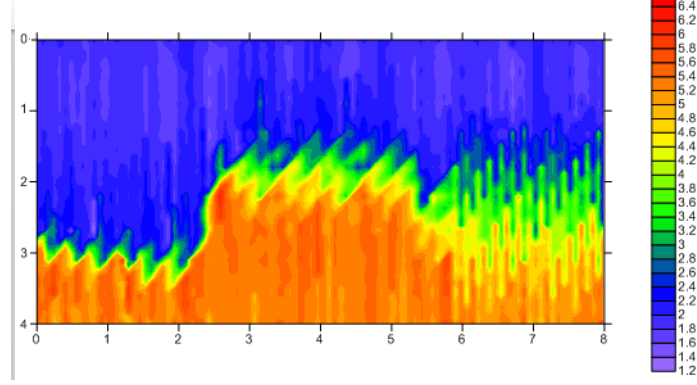

Figura 22 - Modelo $M_{I I}$ parametrizado por ondaleta Haar utilizando-se o filtro passa-alta com ponto de corte igual a $10^{-1.35}$

\section{Discussão e Conclusões}

A representação de um modelo geológico por parametrização utilizando série de ondaleta Haar mostrou-se bastante eficiente, pois, manteve as principais feições dos modelos geológicos considerados e seus respectivos campos de velocidades. Entretanto, sabe-se que uma representação perfeita do campo só é possível se forem considerados todos os valores dos índices $j$ e $k$, isto é: seria necessário fazer os índices $j \mathrm{e}$ $k$ percorrer todos os valores inteiros entre $-\infty$ a $+\infty$, o que é impraticável.

Como a parametrização do campo sem filtragem apresentou um grande numero de coeficientes, fez-se necessário aplicar um filtro do tipo passa-alta para que um número ótimo de coeficientes fosse usado para representar o campo. Usando uma técnica empíricocomputacional, é possível encontrar um ponto de corte que permite reduzir o número de coeficiente sem perda significativa de acurácia. Isto é, preservar de modo reconhecível as principais feições do campo com o menor número possível de coeficientes na série ondaleta.

\section{Agradecimentos}

Agradecemos ao CPGG-UFBA e a PETROBRAS pelo oferecimento de condições para a realização deste trabalho.

\section{Referências}

Morettin, P.A.; 1999. Ondas e Ondaletas: da Análise de Fourier à Análise de Ondaletas. EDUSP.

Teixeira, W.; Toledo, M.C.M.; Fairchild, T.R. \& Taioli, F.; 2000. Decifrando a Terra. $1^{\text {a }}$ Edição.

Araripe, P.T, 2002. Bacia do Recôncavo. Apresentação na Quarta Rodada de Licitações. Superintendência de Definição de Blocos. 